\title{
THE LATTICE OF VARIETIES OF IMPLICATION SEMIGROUPS
}

\author{
SERGEY V. GUSEV, HANAMANTAGOUDA P. SANKAPPANAVAR, \\ AND BORIS M. VERNIKOV
}

\begin{abstract}
An implication semigroup is an algebra of type $(2,0)$ with a binary operation $\rightarrow$ and a 0 -ary operation 0 satisfying the identities $(x \rightarrow y) \rightarrow z \approx$ $x \rightarrow(y \rightarrow z),(x \rightarrow y) \rightarrow z \approx\left[\left(z^{\prime} \rightarrow x\right) \rightarrow(y \rightarrow z)^{\prime}\right]^{\prime}$ and $0^{\prime \prime} \approx 0$ where $\mathbf{u}^{\prime}$ means $\mathbf{u} \rightarrow 0$ for any term $\mathbf{u}$. We completely describe the lattice of varieties of implication semigroups. It turns out that this lattice is non-modular and consists of 16 elements.
\end{abstract}

\section{INTRODUCTION AND SUMMARY}

In the article [9], the second author introduced and examined a new type of algebras as a generalization of De Morgan algebras. These algebras are of type $(2,0)$ with a binary operation $\rightarrow$ and a 0 -ary operation 0 satisfying the identities

$$
\begin{aligned}
& (x \rightarrow y) \rightarrow z \approx\left(\left(z^{\prime} \rightarrow x\right) \rightarrow(y \rightarrow z)^{\prime}\right)^{\prime}, \\
& 0^{\prime \prime} \approx 0
\end{aligned}
$$

where $\mathbf{u}^{\prime}$ means $\mathbf{u} \rightarrow 0$ for any term $\mathbf{u}$. Such algebras are called implication zroupoids. We refer an interested reader to [9] for detailed explanation of the background and motivations.

The class of all implication zroupoids is a variety denoted by IZ. It seems very natural to examine the lattice of its subvarieties. One of the important and interesting subvarieties of $\mathbf{I Z}$ is the class of all associative implication zroupoids, that is algebras from IZ satisfying the identity

$$
(x \rightarrow y) \rightarrow z \approx x \rightarrow(y \rightarrow z) .
$$

It is natural to call such algebras implication semigroups. The class IS of all implication semigroups forms a subvariety in IZ. This subvariety was implicitly mentioned in [9, Lemma 8.21] and investigated more explicitly in the articles [3-5]. (Incidentally, we should mention here that implication zroupoids are referred to as "implicator groupoids" in [4].) But only the location of IS in the subvariety lattice of the variety IZ and "interaction" of IS with other varieties from this lattice were studied in those articles. The aim of this paper is to examine the lattice of subvarieties of the variety IS. Our main result gives a complete description of this lattice.

For convenience of our considerations, we turn to the notation generally accepted in the semigroup theory. As usual, we denote the binary operation by the absence of a symbol, rather than by $\rightarrow$. Since this operation is associative, we will, as a rule, omit brackets in terms. Besides that, the notation 0 for the 0 -ary operation seems to be inappropriate in the framework of examination of implication semigroups, because it is associated with the operation of fixing the zero element in a semigroup with zero. For this reason, we will denote the 0 -ary operation by the symbol $\omega$

2010 Mathematics Subject Classification. Primary 06E75, secondary 08B15.

Key words and phrases. Implication semigroup, variety, lattice of varieties.

The first and the third author were partially supported by the Ministry of Education and Science of the Russian Federation (project 1.6018.2017/8.9) and by the Russian Foundation for Basic Research (grant No. 17-01-00551). 
which does not have any predefined a priori meaning. In this notation, implication semigroups are defined by the associative law $(x y) z \approx x(y z)$ and the following two identities:

$$
\begin{aligned}
x y z & \approx z \omega x y z \omega^{2}, \\
\omega^{3} & \approx \omega .
\end{aligned}
$$

To formulate the main result of the article, we need some notation. As usual, elements of the free implication semigroup over a countably infinite alphabet are called words, while elements of this alphabet are called letters. Words rather than letters are written in bold. We connect two sides of identities by the symbol $\approx$. We denote by $\mathbf{T}$ the trivial variety of implication semigroups. The variety of implication semigroups given (within IS) by the identity system $\Sigma$ is denoted by $\operatorname{var} \Sigma$. Let us fix notation for the following concrete varieties:

$$
\begin{aligned}
& \mathbf{B}:=\operatorname{var}\left\{x \approx x^{2}\right\}, \\
& \mathbf{K}:=\operatorname{var}\left\{x y z \approx x^{2} \approx \omega, x y \approx y x\right\}, \\
& \mathbf{L}:=\operatorname{var}\left\{x y z \approx x^{2} \approx \omega\right\}, \\
& \mathbf{M}:=\operatorname{var}\{x y z \approx \omega, x y \approx y x\}, \\
& \mathbf{N}:=\operatorname{var}\{x y z \approx \omega\}, \\
& \mathbf{S L}:=\operatorname{var}\left\{x \approx x^{2}, x y \approx y x\right\}, \\
& \mathbf{Z M}:=\operatorname{var}\{x y \approx \omega\} .
\end{aligned}
$$

The lattice of all varieties of implication semigroups is denoted by $\mathbb{I S}$.

The main result of the article is the following

Theorem 1.1. The lattice $\mathbb{I S}$ has the form shown in Fig. 1.

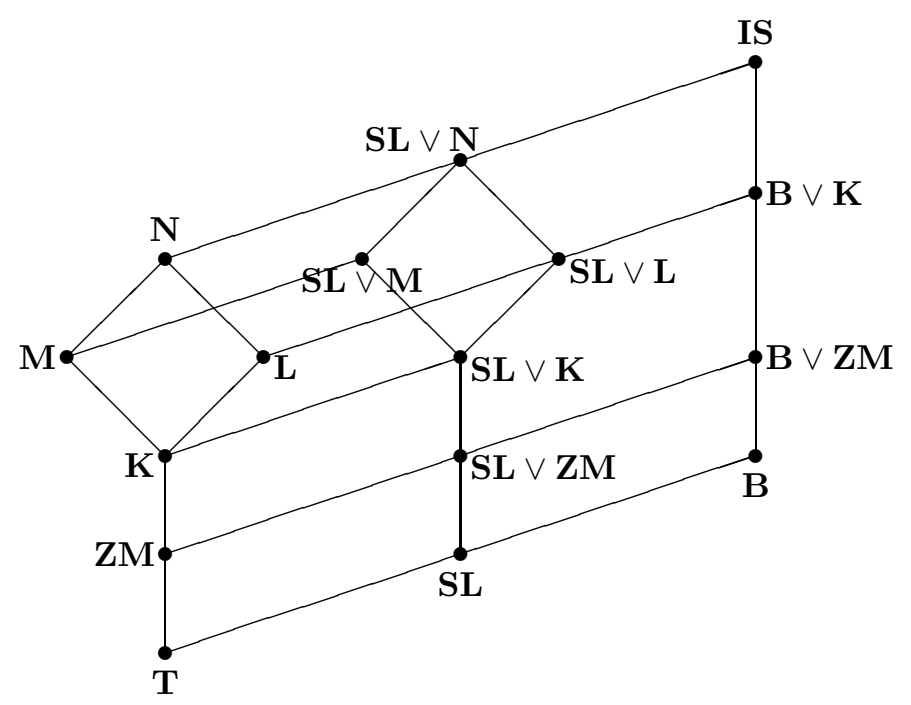

FiguRE 1. The lattice $\mathbb{I S}$

In [9, Problem 5], the second author formulated the question of whether the lattice of all varieties of implication zroupoids is distributive. The following assertion immediately follows from Fig. 1 and provides the negative answer to this question.

Corollary 1.2. The lattice $\mathbb{I S}$ is non-modular. 
This article consists of three sections and two appendixes. Section 2 is devoted to the proof of Theorem 1.1, while Section 3 contains several open problems. The proof of Theorem 1.1 given in Section 2 is based in essential degree on suggestions of the referee on the journal version of the article. The original version of the proof due to the authors is set forth in Appendix A. Finally, Appendix B contains some assertions concerning with one of the problems posed in Section 3.

\section{Proof of the MAIN Result}

To verify Theorem 1.1, we need a few auxiliary assertions. If $\mathbf{u}$ and $\mathbf{v}$ are words and $\varepsilon$ is an identity then we will write $\mathbf{u} \stackrel{\varepsilon}{\approx} \mathbf{v}$ in the case when the identity $\mathbf{u} \approx \mathbf{v}$ follows from $\varepsilon$.

Lemma 2.1. The variety IS satisfies the following identities:

$$
\begin{aligned}
\omega^{2} & \approx \omega, \\
\omega x & \approx x \omega, \\
x y z & \approx x y z \omega .
\end{aligned}
$$

Proof. The following three chains of identities provide deductions of the identities (2.1)-(2.3):

$$
\begin{gathered}
(2.1): \quad \omega^{2} \stackrel{(1.4)}{\approx} \omega^{10}=\omega^{2} \omega \omega \omega^{2} \omega^{2} \omega^{2} \stackrel{(1.3)}{\approx} \omega \omega^{2} \omega^{2}=\omega^{5} \stackrel{(1.4)}{\approx} \omega, \\
(2.2): \quad \omega x \stackrel{(2.1)}{\approx} \omega \omega x \stackrel{(1.3)}{\approx} x \omega \omega \omega x \omega^{2} \stackrel{(2.1)}{\approx}\left(x \omega \omega \omega x \omega^{2}\right) \omega \\
\stackrel{(2.1)}{\approx}(\omega \omega x) \omega \approx \omega \omega x \omega \omega \omega^{2} \stackrel{(1.3)}{\approx} x \omega \omega \stackrel{(2.1)}{\approx} x \omega, \\
(2.3): \quad x y z \stackrel{(1.3)}{\approx} z \omega x y z \omega^{2} \stackrel{(2.1)}{\approx}\left(z \omega x y z \omega^{2}\right) \omega \stackrel{(1.3)}{\approx}(x y z) \omega .
\end{gathered}
$$

Lemma is proved.

An idempotent $e$ of a semigroup $S$ that commutes with every element in $S$ is said to be a central idempotent. The identities (2.1) and (2.2) show that if $S$ is an implication semigroup then the distinguished element $\omega$ of $S$ is a central idempotent. This explains our interest in the following assertion which is a part of semigroup folklore. We provide its proof here for the sake of completeness.

Lemma 2.2. If $e$ is a central idempotent of a semigroup $S$ then $S$ is a subdirect product of its ideal $e S$ and the Rees quotient $S / e S$.

Proof. Clearly, $e S$ is an ideal of $S$ and the natural homomorphism $\eta: S \rightarrow S / e S$ has the property that $\eta(x)=\eta(y)$ if and only if either $x=y$ or $x, y \in e S$. On the other hand, the map $\varphi: S \rightarrow e S$ given by the rule $\varphi(x)=e x$ is a homomorphism of $S$ onto $e S$ and $e x=e y$ implies $x=y$ for $x, y \in e S$. Therefore, if $x, y \in S$ are such that $\eta(x)=\eta(y)$ and $\varphi(x)=\varphi(y)$, then $x=y$. We see that $\varphi$ and $\eta$ are surjective homomorphisms from $S$ onto $e S$ and $S / e S$ respectively, and the intersection of kernels of these homomorphisms is the equality relation. Hence $S$ is a subdirect product of $e S$ and $S / e S$.

Recall that a semigroup is called a band if it satisfies the identity $x^{2} \approx x$. We call a variety of implication semigroups $\mathbf{V}$ a monoid variety if the identities $x \omega \approx \omega x \approx x$ hold in $\mathbf{V}$. Obviously, this means that every semigroup in $\mathbf{V}$ has an identity element and the operation $\omega$ fixes just this element in each semigroup from $\mathbf{V}$.

Lemma 2.3. A variety of implication semigroups is a monoid variety if and only if it is a variety of bands. 
Proof. Any monoid variety satisfies the identities $x \approx \omega^{2} x \stackrel{(1.3)}{\approx} x \omega^{3} x \omega^{2} \approx x^{2}$, while any variety of bands satisfies the identities $\omega x \stackrel{(2.2)}{\approx} x \omega \approx x^{3} \omega \stackrel{(2.3)}{\approx} x^{3} \approx x$.

Lemma 2.4. If $\mathbf{V}$ is an implication semigroup variety then $\mathbf{V}=(\mathbf{V} \wedge \mathbf{B}) \vee(\mathbf{V} \wedge \mathbf{N})$.

Proof. We can assume that the variety $\mathbf{V}$ is generated by an implication semigroup $S$. In view of Lemmas 2.1 and 2.2, the set $\omega S$ is an ideal of $S$ and $S$ is a subdirect product of $\omega S$ and the Rees quotient $S / \omega S$. Clearly, $\omega S$ is an implication semigroup with the distinguished element $\omega$ and $\omega x=x \omega=x$ for every $x \in \omega S$. Then $\omega S \in \mathbf{B}$ by Lemma 2.3. Note also that $S / \omega S$ is an implication semigroup with the distinguished element $\omega S$ and $x y z \stackrel{(2.3)}{=} x y z \omega \stackrel{(2.2)}{=} \omega x y z \in \omega S$ for every $x, y, z \in S$. This implies that $S / \omega S$ satisfies the identity $x y z \approx \omega$ and therefore, is contained in the variety $\mathbf{N}$. Thus, we have proved that $S$ is a subdirect product of the implication semigroups $\omega S \in \mathbf{B}$ and $S / \omega S \in \mathbf{N}$. This implies the required conclusion.

As usual, we denote by $L(\mathbf{X})$ the subvariety lattice of the variety $\mathbf{X}$.

Proof of Theorem 1.1. According to Lemma 2.3, $\mathbf{B}$ is a monoid variety. Therefore, it satisfies the identities $x y x \approx x \omega y x \omega^{2} \stackrel{(1.3)}{\approx} \omega y x \approx y x$. The lattice of varieties of band monoids is completely described in [12]. In view of [12, Proposition 4.7], the lattice $L(\mathbf{B})$ is the 3-element chain $\mathbf{T} \subset \mathbf{S L} \subset \mathbf{B}$.

The variety $\mathbf{N}$ satisfies the identities $\omega x \stackrel{(2.2)}{\approx} x \omega \stackrel{(2.1)}{\approx} x \omega^{2} \approx \omega$. Hence every semigroup from $\mathbf{N}$ contains the zero element and the operation $\omega$ fixes just this element in each semigroup from $\mathbf{N}$. This means that $\mathbf{N}$ is nothing but the variety of all 3-nilpotent semigroups. The subvariety lattice of this variety has the form shown in Fig. 1. This claim can be easily verified directly and is a part of semigroup folklore. It is known at least from the beginning of 1970's (see [7], for instance).

Recall that commutative bands are called semilattices. We fix notation for the following semigroups:

$$
\begin{aligned}
A & :=\{0,1\} \text { - the 2-element semilattice, } \\
B & :=\left\langle e, f, 1 \mid e f=f^{2}=f, f e=e^{2}=e\right\rangle=\{e, f, 1\} \\
K & :=\left\langle a, b, 0 \mid a b=b a, a^{2}=b^{2}=0\right\rangle=\{a, b, a b, 0\} \\
L & :=\left\langle a, b, 0 \mid b a=a^{2}=b^{2}=0\right\rangle=\{a, b, a b, 0\} \\
M & :=\left\langle a, b, 0 \mid a b=b a, a^{2}=a b^{2}=b^{3}=0\right\rangle=\left\{a, b, b^{2}, a b, 0\right\} \\
Z & :=\left\langle a, 0 \mid a^{2}=0\right\rangle=\{a, 0\}
\end{aligned}
$$

where 0 and 1 have the usual sense in semigroup context (the zero element of a semigroup and the identity one, respectively). All these semigroups can be considered as implication semigroups. Indeed, it is easy to see that putting $\omega=1$ in $A, B$ and $\omega=0$ in $K, L, M, Z$, we achieve the fulfillment of the identities (1.3) and (1.4). The variety generated by an implication semigroup $S$ is denoted by var $S$. It is well known and easily verified that $\mathbf{B}=\operatorname{var} B, \mathbf{K}=\operatorname{var} K, \mathbf{L}=\operatorname{var} L, \mathbf{M}=\operatorname{var} M$, $\mathbf{S L}=\operatorname{var} A$ and $\mathbf{Z M}=\operatorname{var} Z$.

Now we are going to prove that the lattice $L(\mathbf{S L} \vee \mathbf{N})$ has the form shown in Fig. 1. Clearly, the implication semigroups $A, L$ and $M$ satisfy the identity $x y \omega \approx y x \omega$. So, this identity holds in $\mathbf{S L} \vee \mathbf{N}$. Since it is false in $B$, we have that $(\mathbf{S L} \vee \mathbf{N}) \wedge \mathbf{B}=$ SL. This fact and Lemma 2.4 imply that $\mathbf{V}=(\mathbf{V} \wedge \mathbf{S L}) \vee(\mathbf{V} \wedge \mathbf{N})$ for every subvariety $\mathbf{V}$ of $\mathbf{S L} \vee \mathbf{N}$. Then $\mathbf{S L} \vee \mathbf{N}$ has at most 12 subvarieties, namely, the ones shown in Fig. 1. We need to verify that these subvarieties are different from each other. For a class $\mathbf{X}$ of implication semigroups, let $\overline{\mathbf{X}}$ stand for the class of all semigroup reducts of implication semigroups in $\mathbf{X}$. Since $\omega \approx x^{3}$ in $\mathbf{N}$, we see that $\overline{\mathbf{V}}$ is a subvariety of $\overline{\mathbf{N}}$ whenever $\mathbf{V}$ is a subvariety of $\mathbf{N}$. Now let $\mathbf{V}$ and $\mathbf{W}$ be two 
different subvarieties of $\mathbf{N}$. Then the semigroup varieties $\overline{\mathbf{V}}$ and $\overline{\mathbf{W}}$ are different as well. It is well known that the semigroup variety $\overline{\mathbf{S L}}$ of all semilattices constitutes a neutral element of the lattice of all semigroup varieties (it is proved explicitly in [11, Proposition 4.1]), whence $\overline{\mathbf{V}} \vee \overline{\mathbf{S L}} \neq \overline{\mathbf{W}} \vee \overline{\mathbf{S L}}$. An identity $\mathbf{u} \approx \mathbf{v}$ is called a semigroup identity if both the words $\mathbf{u}$ and $\mathbf{v}$ do not contain the symbol of 0-ary operation. Any semigroup identity that differentiates $\overline{\mathbf{V}} \vee \overline{\mathbf{S L}}$ from $\overline{\mathbf{W}} \vee \overline{\mathbf{S L}}$ will also differentiate the implication semigroup varieties $\mathbf{V} \vee \mathbf{S L}$ and $\mathbf{W} \vee \mathbf{S L}$.

Further, we are going to prove that the lattice $L(\mathbf{B} \vee \mathbf{Z M})$ has the form shown in Fig. 1. First of all, we note that the identity $x y \approx x y \omega$ holds in $B$ and $Z$ but fails in $K$. Therefore, $(\mathbf{B} \vee \mathbf{Z M}) \wedge \mathbf{N}=\mathbf{Z M}$. This fact and Lemma 2.4 imply that $\mathbf{V}=(\mathbf{V} \wedge \mathbf{B}) \vee(\mathbf{V} \wedge \mathbf{Z M})$ for every subvariety $\mathbf{V}$ of $\mathbf{B} \vee \mathbf{Z M}$. Then $\mathbf{B} \vee \mathbf{Z M}$ has at most 6 subvarieties, namely, the ones shown in Fig. 1. We need to verify that these subvarieties are different from each other. In view of the observations made in the first, the second and the fourth paragraphs of the proof of Theorem 1.1, it remains to show that $\mathbf{S L} \vee \mathbf{Z M} \subset \mathbf{B} \vee \mathbf{Z M}$. This follows from the fact that the identity $x y \approx y x \omega$ holds in $\mathbf{S L} \vee \mathbf{Z M}$ but fails in $\mathbf{B}$.

Lemma 2.4 with $\mathbf{V}=\mathbf{I S}$ implies that $\mathbf{I S}=\mathbf{B} \vee \mathbf{N}$. Since $\mathbf{B}$ has exactly 3 subvarieties and $\mathbf{N}$ has exactly 6 ones, we have that IS has at most 18 subvarieties. Now we aim to show that $\mathbf{B} \vee \mathbf{K}=\mathbf{B} \vee \mathbf{L}$ and $\mathbf{B} \vee \mathbf{M}=\mathbf{B} \vee \mathbf{N}$. The subset $I=\{(e, 0),(f, 0),(1,0)\}$ of the direct product $B \times K$ forms an ideal of $B \times K$. The Rees quotient $(B \times K) / I$ is a 3 -nilpotent implication semigroup that satisfies the identity $x^{2} \approx \omega$ but violates the commutative law. Indeed, $(e, a)(f, b)=(f, a b) \neq(e, a b)=(f, b)(e, a)$. We see that $(B \times K) / I$ lies in $\mathbf{L}$ but does not lie in $\mathbf{K}$. Note that $\mathbf{K}$ is the only maximal subvariety of $\mathbf{L}$. Whence $(B \times K) / I$ generates the variety $\mathbf{L}$. Since $(B \times K) / I \in \mathbf{B} \vee \mathbf{K}$, we have that $\mathbf{L} \subseteq \mathbf{B} \vee \mathbf{K}$. We conclude that $\mathbf{B} \vee \mathbf{L} \subseteq \mathbf{B} \vee \mathbf{K}$, and the converse inclusion is clear. Thus $\mathbf{B} \vee \mathbf{K}=\mathbf{B} \vee \mathbf{L}$. Further, $\mathbf{B} \vee \mathbf{M} \supseteq \mathbf{B} \vee \mathbf{K}=\mathbf{B} \vee \mathbf{L}$. Therefore, $\mathbf{L} \subseteq \mathbf{B} \vee \mathbf{M}$, whence $\mathbf{N}=\mathbf{M} \vee \mathbf{L} \subseteq \mathbf{B} \vee \mathbf{M}$. We get that $\mathbf{B} \vee \mathbf{N} \subseteq \mathbf{B} \vee \mathbf{M}$. The converse inclusion is clear, whence $\mathbf{B} \vee \mathbf{M}=\mathbf{B} \vee \mathbf{N}$. Thus, we have proved that IS has at most 16 subvarieties, namely, the ones shown in Fig. 1. We need to verify that these subvarieties are different from each other. In view of what is said in the fourth and the fifth paragraphs of the proof of Theorem 1.1, it remains to show that $\mathbf{B} \vee \mathbf{K} \subset \mathbf{I S}$. This follows from the above-mentioned equalities $\mathbf{I S}=\mathbf{B} \vee \mathbf{N}=\mathbf{B} \vee \mathbf{M}$ and the fact that the identity $x \omega \approx x^{2}$ holds in $K$ and $B$ but fails in $M$.

\section{OPEN PROBLEMS}

We denote by $\mathbb{Z} \mathbb{Z}$ the lattice of all varieties of implication zroupoids. Theorem 1.1 shows that the lattice $\mathbb{I} \mathbb{Z}$ is non-modular but the following problem still remains open.

Problem 3.1. Determine whether the lattice $\mathbb{I Z}$ satisfies any non-trivial lattice identity.

Recall that a lattice $\langle L ; \vee, \wedge\rangle$ with the least element 0 is called 0 -distribuive if it satisfies the implication

$$
\forall x, y, z \in L: \quad x \wedge z=y \wedge z=0 \longrightarrow(x \vee y) \wedge z=0 .
$$

Lattices of varieties of all classical types of algebras (groups, semigroups, rings, lattices etc.) are well-known to be 0-distributive. The following question seems to be interesting.

Problem 3.2. Determine whether the lattice $\mathbb{I} Z$ is 0 -distributive.

This problem is closely related to knowing the set of all atoms of the lattice $\mathbb{I Z}$. This set is known but not yet published. Indeed, it is well known that any nontrivial variety of algebras contains a simple algebra, i.e. algebra without congruences 
except the trivial and the universal ones (see [1, Theorem 10.13], for instance). The complete list of simple implication zroupoids is provided by [2, Theorem 5.8]. The variety generated by one of these algebras contains either $\mathbf{Z M}$ or $\mathbf{S L}$ or the variety BA of all Boolean algebras. On the other hand, it is easy to see that these three varieties are atoms of $\mathbb{I Z}$. Combining these observations, we have the following

Remark 3.3. The varieties $\mathbf{Z M}$, SL and $\mathbf{B A}$ are the only atoms of the lattice $\mathbb{I}$.

Returning to Problem 3.2, it is easy to see that this problem is equivalent to the following claim: if $\mathbf{A}$ is an atom of the lattice $\mathbb{I} \mathbb{Z}$ and $\mathbf{X}, \mathbf{Y}$ are varieties of implication zroupoids with $\mathbf{X}, \mathbf{Y} \nsupseteq \mathbf{A}$ then $\mathbf{X} \vee \mathbf{Y} \nsupseteq \mathbf{A}$. We have a proof of this fact in the case when $\mathbf{A}$ is one of the varieties $\mathbf{S L}$ or BA (see Appendix B). But the case when $\mathbf{A}=\mathbf{Z M}$ still remains open.

An element $x$ of a lattice $L$ is called neutral if, for any $y, z \in L$, the elements $x$, $y$ and $z$ generate a distributive sublattice of $L$. Neutral elements play an important role in the lattice theory. If $a$ is a neutral element of a lattice $L$ then $L$ is a subdirect product of the principal ideal and the principal filter of $L$ generated by $a$ (see [6, proof of Theorem 254]). So, the knowledge of the set of neutral elements of a lattice gives significant and important information about the structure of this lattice. Fig. 1 shows that the varieties $\mathbf{S L}$ and $\mathbf{Z M}$ are neutral elements of the lattice $\mathbb{I S}$. The following problem seems to be very interesting.

Problem 3.4. Determine whether SL, ZM and BA are neutral elements of the lattice $\mathbb{I} \mathbb{Z}$.

Note that the varieties of all semilattices and of all semigroups with zero multiplication considered as simply semigroup varieties are neutral elements of the lattice of all semigroup varieties (see [11, Proposition 4.1] or Theorem 3.4 in the survey [10]).

\section{APPEndiX A. An Alternative proof of Theorem 1.1}

Here we give an alternative proof of Theorem 1.1. This appendix is divided into 7 subsections.

A.1. Preliminaries. We need a number of auxiliary results.

Lemma A.1. The variety IS satisfies the following identities:

$$
\begin{aligned}
& x y x \approx y x \omega, \\
& x^{2} y \approx x y \omega .
\end{aligned}
$$

Proof. The following two chains of identities provide deductions of the identities (A.1) and (A.2):

$$
\begin{aligned}
& (\mathrm{A} .1): x y x \stackrel{(2.3)}{\approx} x y x \omega^{4} \stackrel{(2.2)}{\approx} x \omega \omega y x \omega^{2} \stackrel{(1.3)}{\approx} \omega y x \stackrel{(2.2)}{\approx} y x \omega, \\
& (\mathrm{A} .2): x^{2} y \stackrel{(2.3)}{\approx} x^{2} y \omega \stackrel{(2.2)}{\approx} x \omega x y \stackrel{(\mathrm{A} .1)}{\approx} \omega x \omega y \stackrel{(2.2)}{\approx} \omega^{2} x y \stackrel{(2.1)}{\approx} \omega x y \stackrel{(2.2)}{\approx} x y \omega .
\end{aligned}
$$

Lemma is proved.

A word that does not contain the symbol $\omega$ is called a semigroup word. The last occurrence sequence of a word $\mathbf{w}$, denoted by $\operatorname{los}(\mathbf{w})$, is the semigroup word obtained from $\mathbf{w}$ by retaining only the last occurrence of each letter. If $\mathbf{w}$ does not contain letters (i.e., if $\mathbf{w}=\omega^{n}$ for some natural number $n$ ) then $\operatorname{los}(\mathbf{w})$ is the empty word. The length of a semigroup word $\mathbf{w}$ is denoted by $\ell(\mathbf{w})$. If a word $\mathbf{w}$ contains the symbol $\omega$ then we put $\ell(\mathbf{w})=\infty$. We denote by $\operatorname{con}(\mathbf{w})$ the content of the word $\mathbf{w}$, i.e. the set of all letters occurring in $\mathbf{w}$.

Corollary A.2. If $\mathbf{w}$ is a word of a length $\geq 3$ then the variety IS satisfies the identity

$$
\mathbf{w} \approx \operatorname{los}(\mathbf{w}) \omega .
$$


Proof. Since $\ell(\mathbf{w}) \geq 3$, the identity (2.3) allows us to assume that $\mathbf{w}$ is not a semigroup word. Further, in view of the identity (2.2), we can assume that $\mathbf{w}=\mathbf{w}^{\prime} \omega$ for some word $\mathbf{w}^{\prime}$. The identities (A.1) and (A.2) permit to delete from $\mathbf{w}$ all but last occurrences of all letters. Therefore, we can assume that every letter from $\operatorname{con}(\mathbf{w})$ occurs in $\mathbf{w}$ at most one time. Finally, the identities (2.1) and (2.2) allow us to delete from $\mathbf{w}$ all but last occurrences of the symbol $\omega$. Therefore, the identity (A.3) holds in IS.

Note that the identities (2.1)-(2.3), (A.1) and (A.2) are particular instances of the identity (A.3).

Corollary A.3. If $\mathbf{u}$ and $\mathbf{v}$ are words such that $\ell(\mathbf{u}), \ell(\mathbf{v}) \geq 3$ and $\operatorname{los}(\mathbf{u})=\operatorname{los}(\mathbf{v})$ then the variety $\mathbf{I S}$ satisfies the identity $\mathbf{u} \approx \mathbf{v}$.

Proof. Indeed, we have $\mathbf{u} \stackrel{(\text { A.3) }}{\approx} \operatorname{los}(\mathbf{u}) \omega=\operatorname{los}(\mathbf{v}) \omega \stackrel{(\text { A.3) }}{\approx} \mathbf{v}$.

We need a description of the identities of a few concrete varieties of implication semigroups. We say that a word $\mathbf{w}$ contains a square if $\mathbf{w}=\mathbf{a b}^{2} \mathbf{c}$ for some word $\mathbf{b}$ and some (possibly empty) words a and $\mathbf{c}$.

Lemma A.4. A non-trivial identity $\mathbf{u} \approx \mathbf{v}$ holds in the variety:

(i) $\mathbf{S L}$ if and only if $\operatorname{con}(\mathbf{u})=\operatorname{con}(\mathbf{v})$;

(ii) $\mathbf{B}$ if and only if $\operatorname{los}(\mathbf{u})=\operatorname{los}(\mathbf{v})$;

(iii) $\mathbf{Z M}$ if and only if $\ell(\mathbf{u}), \ell(\mathbf{v}) \geq 2$;

(iv) $\mathbf{K}$ if and only if either $\mathbf{u} \approx \mathbf{v}$ is the commutative law or each of the words $\mathbf{u}$ and $\mathbf{v}$ either contains a square or has a length $\geq 3$;

(v) $\mathbf{L}$ if and only if each of the words $\mathbf{u}$ and $\mathbf{v}$ either contains a square or has a length $\geq 3$

(vi) $\mathbf{M}$ if and only if either $\mathbf{u} \approx \mathbf{v}$ is the commutative law or $\ell(\mathbf{u}), \ell(\mathbf{v}) \geq 3$;

(vii) $\mathbf{N}$ if and only if $\ell(\mathbf{u}), \ell(\mathbf{v}) \geq 3$.

Proof. (i) Lemma 2.3 implies that any identity that holds in the variety SL is equivalent to a semigroup identity. It is well known and can be easily checked that a semigroup identity $\mathbf{u} \approx \mathbf{v}$ holds in the variety of semilattices SL if and only if $\operatorname{con}(\mathbf{u})=\operatorname{con}(\mathbf{v})$. This completes the proof of the assertion (i).

(ii) Lemma 2.3 shows that an arbitrary identity $\mathbf{u} \approx \mathbf{v}$ is equivalent within $\mathbf{B}$ to the identity $\mathbf{u} \omega \approx \mathbf{v} \omega$. This allows us to consider only identities, both sides of which have a length $\geq 3$. Corollary A.2 allows us now to delete all but last occurrences of any letter in any word. Furthermore, Lemma 2.3 means that we can delete all occurrences of the symbol $\omega$ in every word. This proves the claim (ii).

(iii)-(vii) These assertions are evident.

Lemma A.5. Let $\mathbf{V}$ be a variety of implication semigroups.

(i) If $\mathbf{V} \nsupseteq \mathbf{B}$ then $\mathbf{V}$ satisfies the identity

$$
x y \omega \approx y x \omega .
$$

(ii) If $\mathbf{V} \nsupseteq \mathbf{K}$ then $\mathbf{V}$ satisfies the identity

$$
x y \approx x y \omega .
$$

(iii) If $\mathbf{V} \nsupseteq \mathbf{N}$ then $\mathbf{V}$ satisfies either the commutative law or the identity

$$
x \omega \approx x^{2} .
$$

Proof. Lemma A.4(i) implies that the identities (A.4)-(A.6) hold in the variety SL. Whence, any of these identities is valid in a variety $\mathbf{V}$ if and only if it is valid in $\mathbf{V} \vee \mathbf{S L}$. Further, if $\mathbf{V} \vee \mathbf{S L} \nsupseteq \mathbf{X}$ where $\mathbf{X}$ is one of the varieties $\mathbf{B}, \mathbf{K}$ or $\mathbf{N}$ then 
$\mathbf{V} \nsupseteq \mathbf{X}$. These observations allow us to assume that $\mathbf{V} \supseteq \mathbf{S L}$. Then Lemma A.4(i) applies and we conclude that if $\mathbf{V}$ satisfies an identity $\mathbf{u} \approx \mathbf{v}$ then

$$
\operatorname{con}(\mathbf{u})=\operatorname{con}(\mathbf{v}) \text {. }
$$

(i) By the hypothesis, there is an identity $\mathbf{u} \approx \mathbf{v}$ that is true in $\mathbf{V}$ but fails in B. Lemma A.4(ii) implies that $\operatorname{los}(\mathbf{u}) \neq \operatorname{los}(\mathbf{v})$. If one of the words $\mathbf{u}$ or $\mathbf{v}$ has a length $\leq 2$ then we multiply the identity $\mathbf{u} \approx \mathbf{v}$ by $x^{2}$ for some $x \in \operatorname{con}(\mathbf{u})$ from the left. Clearly, this does not change the words $\operatorname{los}(\mathbf{u})$ and $\operatorname{los}(\mathbf{v})$. Both the sides of the resulting identity have a length $\geq 3$. Thus we can assume without loss of generality that $\ell(\mathbf{u}), \ell(\mathbf{v}) \geq 3$. According to Corollary A.2, we can assume also that $\mathbf{u}=\operatorname{los}(\mathbf{u}) \omega$ and $\mathbf{v}=\operatorname{los}(\mathbf{v}) \omega$. Then the equality (A.7) implies that $\operatorname{con}(\operatorname{los}(\mathbf{u}))=\operatorname{con}(\operatorname{los}(\mathbf{v}))$. Whence there are letters $x$ and $y$ such that $x$ precedes $y$ in $\mathbf{u}$ but $y$ precedes $x$ in $\mathbf{v}$. Now we substitute $\omega$ for all letters from con(u) except $x$ and $y$ in the identity $\mathbf{u} \approx \mathbf{v}$. In view of the identities (2.1) and (2.2), the obtained identity implies (A.4).

(ii) By the hypothesis, there is an identity $\mathbf{u} \approx \mathbf{v}$ that is true in $\mathbf{V}$ but fails in K. Lemma A.4(iv) implies that the identity $\mathbf{u} \approx \mathbf{v}$ is not the commutative law and one of the words $\mathbf{u}$ and $\mathbf{v}$, say $\mathbf{u}$, has a length $\leq 2$ and is not a square. If $\ell(\mathbf{u})=1$ then we can multiply the identity $\mathbf{u} \approx \mathbf{v}$ by some letter $y \notin \operatorname{con}(\mathbf{u})$ from the right. Thus we can assume that $\ell(\mathbf{u})=2$, whence $\mathbf{u}=x y$. Further, $\mathbf{v} \neq y x$ because the identity $\mathbf{u} \approx \mathbf{v}$ is not the commutative law. The equality (A.7) implies now that $\ell(\mathbf{v}) \geq 3$. Hence $\mathbf{V}$ satisfies the identities $x y=\mathbf{u} \approx \mathbf{v} \stackrel{(\text { A.3) }}{\approx} \operatorname{los}(\mathbf{v}) \omega$ and therefore, the identity

$$
x y \approx \operatorname{los}(\mathbf{v}) \omega .
$$

If $\operatorname{los}(\mathbf{v})=x y$ then we are done. Otherwise, $\operatorname{los}(\mathbf{v})=y x$ and we have

$$
x y \stackrel{(\mathrm{A} .8)}{\approx} y x \omega \stackrel{(\mathrm{A} .8)}{\approx} x y \omega^{2} \stackrel{(2.1)}{\approx} x y \omega .
$$

(iii) By the hypothesis, there is an identity $\mathbf{u} \approx \mathbf{v}$ that is true in $\mathbf{V}$ but fails in N. Lemma A.4(vii) implies that one of the words $\mathbf{u}$ and $\mathbf{v}$, say $\mathbf{u}$, has a length $\leq 2$. We can assume without loss of generality that $\ell(\mathbf{u}) \leq \ell(\mathbf{v})$. If $\ell(\mathbf{u})=1$ then we can multiply the identity $\mathbf{u} \approx \mathbf{v}$ by some letter $y$ from the right. Thus we can assume that $\ell(\mathbf{u})=2$, whence $\mathbf{u} \in\left\{x y, x^{2}\right\}$. Recall that the equality (A.7) holds.

Suppose at first that $\mathbf{u}=x^{2}$. Then $\ell(\mathbf{v}) \geq 3$ and $\operatorname{los}(\mathbf{v})=x$. Therefore, $\mathbf{V}$ satisfies the identities $x^{2}=\mathbf{u} \approx \mathbf{v} \stackrel{(\text { A.3) }}{\approx} \operatorname{los}(\mathbf{v}) \omega=x \omega$. Whence, the identity (A.6) holds in $\mathbf{V}$.

Finally, let $\mathbf{u}=x y$. If $\mathbf{v}=y x$ then the variety $\mathbf{V}$ is commutative, and we are done. Let now $\mathbf{v} \neq y x$. Then $\ell(\mathbf{v}) \geq 3$. Substituting $x$ for $y$ in $\mathbf{u} \approx \mathbf{v}$, we get the situation considered in the previous paragraph.

Lemma A.6. Let $\mathbf{V}$ be a variety of implication semigroups.

(i) If $\mathbf{V} \nsupseteq \mathbf{S L}$ then $\mathbf{V} \subseteq \mathbf{N}$.

(ii) If $\mathbf{V} \nsupseteq \mathbf{Z M}$ then $\mathbf{V} \subseteq \mathbf{B}$.

Proof. (i) Suppose that SL $\nsubseteq \mathbf{V}$. In view of Lemma A.4(i), V satisfies an identity $\mathbf{u} \approx \mathbf{v}$ with $\operatorname{con}(\mathbf{u}) \neq \operatorname{con}(\mathbf{v})$. We can assume without loss of generality that there is a letter $x \in \operatorname{con}(\mathbf{u}) \backslash \operatorname{con}(\mathbf{v})$. One can substitute $\omega$ to all letters except $x$ in the identity $\mathbf{u} \approx \mathbf{v}$. The identities (2.1) and (2.2) imply that $\mathbf{V}$ satisfies the identity $x^{k} \omega \approx \omega$ for some $k$. Corollary A.3 implies that the variety IS satisfies the identity $x y z \approx(x y z)^{n} \omega$ for any natural $n$. Then the identities $x y z \approx(x y z)^{k} \omega \approx \omega$ hold in $\mathbf{V}$, whence $\mathbf{V} \subseteq \mathbf{N}$.

(ii) Suppose that $\mathbf{Z M} \nsubseteq \mathbf{V}$. It suffices to verify that $\mathbf{V} \vee \mathbf{S L} \subseteq \mathbf{B}$. Whence, we can assume that $\mathbf{V} \supseteq \mathbf{S L}$. In view of Lemma A.4(iii), $\mathbf{V}$ satisfies a non-trivial 
identity of the kind $x \approx \mathbf{v}$. Lemma A.4(i) implies that $\operatorname{con}(\mathbf{v})=\{x\}$. Clearly, $\ell(\mathbf{v})>1$. If $\ell(\mathbf{v})=2$ then $\mathbf{v}=x^{2}$, whence $\mathbf{V} \subseteq \mathbf{B}$. Let now $\ell(\mathbf{v}) \geq 3$. Then Corollary A.3 applies and we conclude that the identity $\mathbf{v}^{2} \approx \mathbf{v}$ is true in the variety IS. Therefore, $\mathbf{V}$ satisfies the identities $x^{2} \approx \mathbf{v}^{2} \approx \mathbf{v} \approx x$, whence $\mathbf{V} \subseteq \mathbf{B}$ again.

A.2. The structure of the lattices $L(\mathbf{B})$ and $L(\mathbf{N})$. According to Lemma 2.3, $\mathbf{B}$ is a monoid variety. Therefore, it satisfies the identities $x y x \stackrel{(\text { A.1) }}{\approx} y x \omega \approx y x$. The lattice of varieties of band monoids is completely described in [12]. In view of [12, Proposition 4.7], the lattice $L(\mathbf{B})$ is the 3-element chain $\mathbf{T} \subset \mathbf{S L} \subset \mathbf{B}$.

The variety $\mathbf{N}$ satisfies the identities $\omega x \stackrel{(2.2)}{\approx} x \omega \stackrel{(2.1)}{\approx} x \omega^{2} \approx \omega$. Hence every semigroup from $\mathbf{N}$ contains the zero element and the operation $\omega$ fixes just this element in each semigroup from $\mathbf{N}$. This means that $\mathbf{N}$ is nothing but the variety of all nilpotent of degree 3 semigroups. The subvariety lattice of this variety has the form shown in Fig. 1. This claim can be easily verified directly and is a part of a semigroup folklore. It is known at least from the beginning of 1970's (see [7], for instance).

A.3. Identity bases for certain varieties. Here we prove the following equalities:

$$
\begin{aligned}
& \mathbf{S L} \vee \mathbf{K}=\operatorname{var}\left\{x \omega \approx x^{2}, x y \approx y x\right\}, \\
& \mathbf{S L} \vee \mathbf{L}=\operatorname{var}\left\{x \omega \approx x^{2}, x y \omega \approx y x \omega\right\}, \\
& \mathbf{S L} \vee \mathbf{M}=\operatorname{var}\{x y \approx y x\}, \\
& \mathbf{S L} \vee \mathbf{N}=\operatorname{var}\{x y \omega \approx y x \omega\}, \\
& \mathbf{S L} \vee \mathbf{Z M}=\operatorname{var}\{x y \approx y x \omega\} .
\end{aligned}
$$

Let $\varepsilon$ be one of these equalities. The fact that the left-hand side of $\varepsilon$ is contained in its right-hand side is evident. One can verify reverse inclusions.

We start with the equality (A.12). Let $\mathbf{u} \approx \mathbf{v}$ be an identity that holds in the variety $\mathbf{S L} \vee \mathbf{N}$. The items (i) and (vii) of Lemma A.4 imply that $\operatorname{con}(\mathbf{u})=\operatorname{con}(\mathbf{v})$ and $\ell(\mathbf{u}), \ell(\mathbf{v}) \geq 3$. Put $\operatorname{con}(\mathbf{u})=\operatorname{con}(\mathbf{v})=\left\{x_{1}, x_{2}, \ldots, x_{n}\right\}$. Then Corollary A.2 allows us to assume that our identity coincides with the identity

$$
x_{1} x_{2} \cdots x_{n} \omega \approx x_{\pi(1)} x_{\pi(2)} \cdots x_{\pi(n)} \omega
$$

for some permutation $\pi$ on the set $\{1,2, \ldots, n\}$. But an arbitrary identity of such a form follows from the identities (2.1), (2.2) and (A.4), whence $\operatorname{var}\{x y \omega \approx y x \omega\} \subseteq$ $\mathbf{S L} \vee \mathbf{N}$. This proves the equality (A.12).

The items (vi) and (vii) of Lemma A.4 imply that a unique identity that holds in $\mathbf{S L} \vee \mathbf{M}$ but fails in $\mathbf{S L} \vee \mathbf{N}$ is the commutative law. In view of what was said in the previous paragraph, this implies the equality (A.11).

Let now $\mathbf{u} \approx \mathbf{v}$ be an arbitrary identity that holds in $\mathbf{S L} \vee \mathbf{L}$. The items (i) and (v) of Lemma A.4 imply that $\operatorname{con}(\mathbf{u})=\operatorname{con}(\mathbf{v})$ and each of the words $\mathbf{u}$ and $\mathbf{v}$ either has a length $\geq 3$ or contains a square. If one of the sides of the identity $\mathbf{u} \approx \mathbf{v}$ contains a square then we can apply the identity (A.6). Both the sides of the obtained identity will have a length $\geq 3$. Thus, we can assume that $\ell(\mathbf{u}), \ell(\mathbf{v}) \geq$ 3. Then the items (i) and (vii) of Lemma A.4 apply with the conclusion that the identity $\mathbf{u} \approx \mathbf{v}$ holds in $\mathbf{S L} \vee \mathbf{N}$. The equality (A.12) implies that $\operatorname{var}\{x \omega \approx$ $\left.x^{2}, x y \omega \approx y x \omega\right\} \subseteq \mathbf{S L} \vee \mathbf{N}$. Therefore, the identity $\mathbf{u} \approx \mathbf{v}$ holds in $\operatorname{var}\{x \omega \approx$ $\left.x^{2}, x y \omega \approx y x \omega\right\}$. Thus, the last variety is contained in $\mathbf{S L} \vee \mathbf{L}$. This proves the equality (A.10).

Let now $\mathbf{u} \approx \mathbf{v}$ be an arbitrary identity that holds in $\mathbf{S L} \vee \mathbf{K}$. Suppose that this identity differs from the commutative law. Comparison of the items (iv) and (v) of Lemma A.4 shows that this identity holds in $\mathbf{S L} \vee \mathbf{L}$. Besides that, the variety 
SL $\vee \mathbf{K}$ is commutative. The equality (A.10) proved in the previous paragraph implies now that the identity $\mathbf{u} \approx \mathbf{v}$ holds in the variety

$$
\operatorname{var}\left\{x \omega \approx x^{2}, x y \omega \approx y x \omega, x y \approx y x\right\}=\operatorname{var}\left\{x \omega \approx x^{2}, x y \approx y x\right\} .
$$

Thus, the last variety is contained in $\mathbf{S L} \vee \mathbf{K}$ in any case. This proves the equality (A.9).

Finally, let $\mathbf{u} \approx \mathbf{v}$ be an identity that holds in $\mathbf{S L} \vee \mathbf{Z M}$. The items (i) and (iii) of Lemma A.4 imply that $\operatorname{con}(\mathbf{u})=\operatorname{con}(\mathbf{v})$ and $\ell(\mathbf{u}), \ell(\mathbf{v}) \geq 2$. Further, the items (i) and (vii) of the same lemma imply that the identity

$$
\mathbf{u} \omega \approx \mathbf{v} \omega
$$

holds in SL $\vee \mathbf{N}$. The equality (A.12) implies that the last identity follows from the identity (A.4). In turn, the last identity follows from the identity

$$
x y \approx y x \omega .
$$

Indeed, if we multiply (A.15) on $\omega$ from the right and use the identity (2.1), we obtain (A.4). Summarizing all we say, we get that the identity (A.14) holds in the variety $\operatorname{var}\{x y \approx y x \omega\}$. Besides that, it is clear that $\operatorname{var}\{x y \approx y x \omega\} \nsupseteq \mathbf{K}$. Lemma A.5(ii) implies that the variety $\operatorname{var}\{x y \approx y x \omega\}$ satisfies the identity (A.5). Hence this variety satisfies the identities $\mathbf{u} \stackrel{(\text { A.5) }}{\approx} \mathbf{u} \omega \stackrel{(\text { A.14) }}{\approx} \mathbf{v} \omega \stackrel{(\text { A.5) }}{\approx} \mathbf{v}$. The equality (A.13) is proved.

A.4. The structure of the lattice $L(\mathbf{S L} \vee \mathbf{Z M})$. Here we aim to verify that the variety $\mathbf{S L} \vee \mathbf{Z M}$ contains only four subvarieties, namely $\mathbf{T}, \mathbf{S L}, \mathbf{Z M}$ and $\mathbf{S L} \vee \mathbf{Z M}$. Let $\mathbf{V} \subset \mathbf{S L} \vee \mathbf{Z M}$. Results of Subsection A.2 imply that $\mathbf{S L}$ and $\mathbf{Z M}$ are minimal non-trivial varieties of implication semigroups. Thus, it suffices to check that $\mathbf{V}$ is contained in one of the varieties SL or $\mathbf{Z M}$. Clearly, either SL $\nsubseteq \mathbf{V}$ or $\mathbf{Z M} \nsubseteq \mathbf{V}$. If $\mathbf{Z M} \nsubseteq \mathbf{V}$ then Lemma A.6(ii) implies that $\mathbf{V} \subseteq \mathbf{B}$. Since the variety $\mathbf{S L} \vee \mathbf{Z M}$ and therefore, $\mathbf{V}$ is commutative, we have that $\mathbf{V} \subseteq \mathbf{S L}$ in this case. Suppose now that $\mathbf{S L} \not \mathbf{V}$. Lemma A.6(i) implies that $\mathbf{V} \subseteq \mathbf{N}$. Suppose that $\mathbf{K} \subseteq \mathbf{V}$. Then $\mathbf{K} \subseteq \mathbf{S L} \vee \mathbf{Z M}$. The equality (A.13) implies then that the identity (A.15) is true in $\mathbf{K}$. But this identity implies within $\mathbf{N}$ the identities $x y \approx y x \omega \approx \omega$ (see the second paragraph in Subsection A.2). The identity $x y \approx \omega$ evidently fails in $\mathbf{K}$, a contradiction. We prove that $\mathbf{V} \subseteq \mathbf{N}$ but $\mathbf{K} \nsubseteq \mathbf{V}$. The description of the lattice $L(\mathbf{N})$ given in Subsection A.2 implies now that $\mathbf{V} \subseteq \mathbf{Z M}$ (see Fig. 1).

A.5. The structure of the lattice $L(\mathbf{S L} \vee \mathbf{N})$. Here we are going to prove that the lattice $L(\mathbf{S L} \vee \mathbf{N})$ has the form shown in Fig. 1. Let $\mathbf{V} \subset \mathbf{S L} \vee \mathbf{N}$. By Lemma A.6(i), the lattice $L(\mathbf{S L} \vee \mathbf{N})$ is the set-theoretical union of the lattice $L(\mathbf{N})$ and the interval $[\mathbf{S L}, \mathbf{S L} \vee \mathbf{N}]$. The lattice $L(\mathbf{N})$ is described in Subsection A.2. It remains to verify that the interval $[\mathbf{S L}, \mathbf{S L} \vee \mathbf{N}]$ has the form shown in Fig. 1. In other words, we need to check that if $\mathbf{S L} \subseteq \mathbf{V} \subset \mathbf{S L} \vee \mathbf{N}$ then $\mathbf{V}$ coincides with one of the varieties $\mathbf{S L}, \mathbf{S L} \vee \mathbf{Z M}, \mathbf{S L} \vee \mathbf{K}, \mathbf{S L} \vee \mathbf{L}$ or $\mathbf{S L} \vee \mathbf{M}$. Since $\mathbf{N} \nsubseteq \mathbf{V}$, Lemma A.5(iii) and the equalities (A.10) and (A.11) imply that $\mathbf{V}$ is contained in one of the varieties $\mathbf{S L} \vee \mathbf{L}$ or $\mathbf{S L} \vee \mathbf{M}$. If $\mathbf{V}$ coincides with one of these varieties then we are done. Let now $\mathbf{V} \neq \mathbf{S L} \vee \mathbf{L}$ and $\mathbf{V} \neq \mathbf{S L} \vee \mathbf{M}$.

Suppose that $\mathbf{S L} \vee \mathbf{K} \subseteq \mathbf{V}$. If $\mathbf{V} \subset \mathbf{S L} \vee \mathbf{L}$ then the variety $\mathbf{V}$ is commutative by the items (iv) and (v) of Lemma A.4. Then the equalities (A.9) and (A.10) show that $\mathbf{V}=\mathbf{S L} \vee \mathbf{K}$. Suppose now that $\mathbf{V} \subset \mathbf{S L} \vee \mathbf{M}$. Then the items (iv) and (vi) of Lemma A.4 imply that $\mathbf{V}$ satisfies a non-trivial identity of the kind $\mathbf{u} \approx \mathbf{v}$ where the word $\mathbf{u}$ contains a square and $\ell(\mathbf{u})<3$. Clearly, $\mathbf{u}=x^{2}$. Thus, $\mathbf{V}$ satisfies a non-trivial identity of the kind $x^{2} \approx \mathbf{v}$. Lemma A.4(i) implies that $\operatorname{con}(\mathbf{v})=\{x\}$. It is clear that $\ell(\mathbf{v}) \neq 2$. If $\ell(\mathbf{v})=1$ then our identity implies $x^{3} \approx x^{2}$. Finally, let $\ell(\mathbf{v}) \geq 3$. Then $\mathbf{V}$ satisfies the identities $x^{2} \approx \mathbf{v} \stackrel{(\text { A..3) }}{\approx} \operatorname{los}(\mathbf{v}) \omega=x \omega$. Besides 
that, $\mathbf{V}$ is commutative because $\mathbf{V} \subseteq \mathbf{S L} \vee \mathbf{M}$. The equality (A.9) implies now that $\mathbf{V}=\mathbf{S L} \vee \mathbf{K}$.

It remains to consider the case when $\mathbf{S L} \vee \mathbf{K} \nsubseteq \mathbf{V}$. Lemma A.5(ii) and the equality (A.12) imply that the identities $x y \stackrel{(\text { A.5) }}{\approx} x y \omega \stackrel{(\text { A.4) }}{\approx} y x \omega$ hold in V. Therefore, $\mathbf{V}$ satisfies the identity (A.15). This fact together with the equality (A.13) imply that $\mathbf{V} \subseteq \mathbf{S L} \vee \mathbf{Z M}$. The result of Subsection A.4 implies now that either $\mathbf{V}=\mathbf{S L} \vee \mathbf{Z M}$ or $\mathbf{V}=\mathbf{S L}$ (see Fig. 1).

A.6. The structure of the lattice $L(\mathbf{B} \vee \mathbf{Z M})$. Here we aim to check that the lattice $L(\mathbf{B} \vee \mathbf{Z M})$ has the form shown in Fig. 1. First of all we prove that

$$
\mathbf{B} \vee \mathbf{Z M}=\operatorname{var}\{x y \approx x y \omega\} .
$$

The inclusion $\mathbf{B} \vee \mathbf{Z M} \subseteq \operatorname{var}\{x y \approx x y \omega\}$ follows from Lemma 2.3 and the definition of the variety $\mathbf{Z M}$. One can verify the inverse inclusion. Let $\mathbf{u} \approx \mathbf{v}$ be an arbitrary identity that holds in $\mathbf{B} \vee \mathbf{Z M}$. The items (ii) and (iii) of Lemma A.4 imply that $\operatorname{los}(\mathbf{u})=\operatorname{los}(\mathbf{v})$ and $\ell(\mathbf{u}), \ell(\mathbf{v}) \geq 2$. Clearly, $\operatorname{los}(\mathbf{u} \omega)=\operatorname{los}(\mathbf{v} \omega)$. Now Corollary A.3 implies that the variety IS satisfies the identity $\mathbf{u} \omega \approx \mathbf{v} \omega$. Then the variety $\operatorname{var}\{x y \approx x y \omega\}$ satisfies the identities $\mathbf{u} \stackrel{(\text { A. } .5)}{\approx} \mathbf{u} \omega \approx \mathbf{v} \omega \stackrel{(\text { A. } .5)}{\approx} \mathbf{v}$, whence $\mathbf{u} \approx \mathbf{v}$ holds in $\operatorname{var}\{x y \approx x y \omega\}$. The equality (A.16) is proved.

Let $\mathbf{V} \subset \mathbf{B} \vee \mathbf{Z M}$. In view of the results of Subsections A.2 and A.4, it remains to verify that either $\mathbf{V} \subseteq \mathbf{B}$ or $\mathbf{V} \subseteq \mathbf{S L} \vee \mathbf{Z M}$. Clearly, either $\mathbf{Z M} \nsubseteq \subseteq \mathbf{V}$ or $\mathbf{B} \nsubseteq \mathbf{V}$. If $\mathbf{Z M} \nsubseteq \mathbf{V}$ then Lemma A.6(ii) implies that $\mathbf{V} \subseteq \mathbf{B}$. Suppose now that $\mathbf{B} \nsubseteq \mathbf{V}$. Then Lemma A.5(i) implies that $\mathbf{V}$ satisfies the identity (A.4). Besides that, the equality (A.16) implies that the identity (A.5) holds in V. Therefore, the identities $\stackrel{(\text { A.5) }}{\approx}$ (A.4)

(A $2 y x \omega$ are satisfied by $\mathbf{V}$. We see that $\mathbf{V}$ satisfies the identity (A.15). This fact and the equality (A.13) implies that $\mathbf{V} \subseteq \mathbf{S L} \vee \mathbf{Z M}$.

A.7. Completion of the proof. First of all, we verify that $\mathbf{I S}=\mathbf{B} \vee \mathbf{N}$. Let $\mathbf{u} \approx \mathbf{v}$ be an arbitrary identity that holds in $\mathbf{B} \vee \mathbf{N}$. The items (ii) and (vii) of Lemma A.4 imply that $\operatorname{los}(\mathbf{u})=\operatorname{los}(\mathbf{v})$ and $\ell(\mathbf{u}), \ell(\mathbf{v}) \geq 3$. Then Corollary A.3 implies that the variety IS satisfies the identity $\mathbf{u} \approx \mathbf{v}$, and we are done.

Now we verify that

$$
\mathbf{B} \vee \mathbf{K}=\operatorname{var}\left\{x \omega \approx x^{2}\right\} .
$$

Lemma 2.3 and the definition of the variety $\mathbf{B}$ imply that this variety satisfies the identity (A.6). The variety $\mathbf{K}$ also satisfies this identity by Lemma A.4(iv). Hence $\mathbf{B} \vee \mathbf{K} \subseteq \operatorname{var}\left\{x \omega \approx x^{2}\right\}$. One can verify the inverse inclusion. Let $\mathbf{u} \approx \mathbf{v}$ be an arbitrary identity that holds in $\mathbf{B} \vee \mathbf{K}$. The items (ii) and (iv) of Lemma A.4 and the fact that the variety $\mathbf{B}$ is non-commutative imply that $\operatorname{los}(\mathbf{u})=\operatorname{los}(\mathbf{v})$ and each of the words $\mathbf{u}$ and $\mathbf{v}$ either has a length $\geq 3$ or contains a square. If a word contains a square then we can apply the identity (A.6) and obtain a word of a length $\geq 3$. Thus, we can assume that $\ell(\mathbf{u}), \ell(\mathbf{v}) \geq 3$. Then Corollary A.3 implies that the identity $\mathbf{u} \approx \mathbf{v}$ holds in the variety IS and therefore, in $\operatorname{var}\left\{x \omega \approx x^{2}\right\}$. The equality (A.17) is proved.

Now we are well prepared to quickly complete the proof of Theorem 1.1. Let $\mathbf{V} \subset \mathbf{I S}$. Since $\mathbf{I S}=\mathbf{B} \vee \mathbf{N}$, either $\mathbf{B} \nsubseteq \mathbf{V}$ or $\mathbf{N} \nsubseteq \mathbf{V}$. In the former case, Lemma A.5(i) and the equality (A.12) imply that $\mathbf{V} \subseteq \mathbf{S L} \vee \mathbf{N}$, while in the latter case $\mathbf{V} \subseteq \mathbf{B} \vee \mathbf{K}$ by Lemma A.4(iv) and the equality (A.17). As we have seen in Subsection A.5, the lattice $L(\mathbf{S L} \vee \mathbf{N})$ has the form shown in Fig. 1. It remains to consider the lattice $L(\mathbf{B} \vee \mathbf{K})$.

Let $\mathbf{V} \subset \mathbf{B} \vee \mathbf{K}$. Then either $\mathbf{B} \nsubseteq \mathbf{V}$ or $\mathbf{K} \nsubseteq \mathbf{V}$. As we have seen in the previous paragraph, $\mathbf{V} \subseteq \mathbf{S L} \vee \mathbf{N}$ in the former case. Then $\mathbf{V} \subseteq(\mathbf{S L} \vee \mathbf{N}) \wedge(\mathbf{B} \vee \mathbf{K})$. Now the equalities (A.10), (A.12) and (A.17) apply with the conclusion that $\mathbf{V} \subseteq \mathbf{S L} \vee \mathbf{L}$. According to results of Subsection A.5, the lattice $L(\mathbf{S L} \vee \mathbf{L})$ has the form shown 
in Fig. 1. Finally, let $\mathbf{K} \nsubseteq \mathbf{V}$. Then Lemma A.5(ii) and the equality (A.16) imply that $\mathbf{V} \subseteq \mathbf{B} \vee \mathbf{Z M}$. It remains to refer to results of Subsection A.6.

Theorem 1.1 is proved.

\section{APPENDIX B. On Problem 3.2}

Here we consider arbitrary varieties of implication zroupoids without assumption that the binary operation is associative. For this reason, we return below to the original notation of the binary and 0 -ary operations by $\rightarrow$ and 0 respectively.

As we have already noted in Section 3, Problem 3.2 is equivalent to the following claim: if $\mathbf{A}$ is one of the varieties $\mathbf{Z M}$, SL or $\mathbf{B A}$, while $\mathbf{X}, \mathbf{Y}$ are varieties of implication zroupoids with $\mathbf{X}, \mathbf{Y} \nsupseteq \mathbf{A}$ then $\mathbf{X} \vee \mathbf{Y} \nsupseteq \mathbf{A}$. Here we prove the following

Proposition B.1. If $\mathbf{A}$ is one of the varieties $\mathbf{S L}$ or $\mathbf{B A}$, while $\mathbf{X}, \mathbf{Y}$ are varieties of implication zroupoids with $\mathbf{X}, \mathbf{Y} \nsupseteq \mathbf{A}$ then $\mathbf{X} \vee \mathbf{Y} \nsupseteq \mathbf{A}$.

Proof. First of all, we consider the following two 2-element implication zroupoids $\mathbf{2}_{\mathrm{s}}$ and $\mathbf{2}_{\mathrm{b}}$ :

$$
\mathbf{2}_{\mathbf{s}}: \begin{array}{r|rr}
\rightarrow & 0 & 1 \\
\hline 0 & 0 & 1 \\
1 & 1 & 1
\end{array} \quad \quad \mathbf{2}_{\mathbf{b}}: \begin{array}{r|rr}
\rightarrow & 0 & 1 \\
\hline 0 & 1 & 1 \\
1 & 0 & 1
\end{array}
$$

It is evident that the algebras $\mathbf{2}_{\mathbf{s}}$ and $\mathbf{2}_{\mathbf{b}}$ generate the varieties $\mathbf{S L}$ and $\mathbf{B A}$ respectively.

First, we shall prove Proposition B.1 with $\mathbf{A}=\mathbf{S L}$. In fact, this claim is well known and can be easily deduced from the known universal-algebraic facts summarized in the survey [8], for instance. However, for the sake of completeness, we prefer to provide here a simple proof not depending on any other results.

Let $\mathbf{X}$ and $\mathbf{Y}$ be varieties of implication zroupoids with $\mathbf{X}, \mathbf{Y} \nsupseteq \mathbf{S L}$. Lemma A.4(i) implies that the variety $\mathbf{X}$ satisfies some identity $\mathbf{a} \approx \mathbf{b}$ such that $\operatorname{con}(\mathbf{a}) \neq$ $\operatorname{con}(\mathbf{b})$. We can assume without less of generality that there is a variable $x$ with $x \in \operatorname{con}(\mathbf{a}) \backslash \operatorname{con}(\mathbf{b})$. One can substitute 0 for all variables occurring in the identity $\mathbf{a} \approx \mathbf{b}$ except $x$. Then we obtain an identity of the form $\mathbf{u}(x) \approx \mathbf{p}$ such that $\operatorname{con}(\mathbf{u})=\{x\}$ and $\operatorname{con}(\mathbf{p})=\varnothing$. Clearly, this identity holds in $\mathbf{X}$. Analogously, the variety $\mathbf{Y}$ satisfies an identity of the form $\mathbf{v}(x) \approx \mathbf{q}$ such that $\operatorname{con}(\mathbf{v})=\{x\}$ and $\operatorname{con}(\mathbf{q})=\varnothing$. It is evident that the variety $\mathbf{X}$ satisfies the identity

$$
\mathbf{v}(\mathbf{u}(x)) \approx \mathbf{v}(\mathbf{p}) \text {. }
$$

Furthermore, substituting $\mathbf{u}(x)$ first, and then $\mathbf{p}$ for $x$ in $\mathbf{v}(x) \approx \mathbf{q}$, we get that the identities $\mathbf{v}(\mathbf{u}(x)) \approx \mathbf{q}$ and $\mathbf{v}(\mathbf{p}) \approx \mathbf{q}$ are satisfied in $\mathbf{Y}$. This means that the identity (B.1) holds in $\mathbf{Y}$ and therefore, in $\mathbf{X} \vee \mathbf{Y}$. Since $\operatorname{con}(\mathbf{v}(\mathbf{u}(x)))=\{x\}$ and $\operatorname{con}(\mathbf{v}(\mathbf{p}))=\varnothing$, we can apply Lemma A.4(i) and conclude that $\mathbf{X} \vee \mathbf{Y} \nsupseteq \mathbf{S L}$. Thus, we have proved Proposition B.1 with $\mathbf{A}=\mathbf{S L}$.

Next, to prove Proposition B.1 with $\mathbf{A}=\mathbf{S L}$. To prove this proposition with $\mathbf{A}=\mathbf{B A}$, we need the following

Lemma B.2. The variety $\mathbf{I Z}$ satisfies the following identities:

$$
\begin{aligned}
& (x \rightarrow y) \rightarrow z \approx\left(\left(0^{\prime} \rightarrow x\right) \rightarrow y\right) \rightarrow z, \\
& (x \rightarrow y) \rightarrow z \approx((x \rightarrow y) \rightarrow z)^{\prime \prime}, \\
& \left((0 \rightarrow x) \rightarrow 0^{\prime}\right) \rightarrow y \approx\left(x \rightarrow 0^{\prime}\right) \rightarrow y, \\
& 0^{\prime} \rightarrow 0^{\prime} \approx 0^{\prime} \\
& 0 \rightarrow 0^{\prime} \approx 0^{\prime}
\end{aligned}
$$


Proof. The identities (B.2) and (B.3) were found in [9, Lemma 7.5(d)] and [4, Lemma 2.8(2)] respectively. This is the deduction of the identity (B.4):

$$
\begin{aligned}
\left((0 \rightarrow x) \rightarrow 0^{\prime}\right) \rightarrow y & \stackrel{(1.1)}{\approx}\left(\left(0^{\prime \prime} \rightarrow 0\right) \rightarrow\left(x \rightarrow 0^{\prime}\right)^{\prime}\right)^{\prime} \rightarrow y \\
& \stackrel{(1.2)}{\approx}\left(0^{\prime} \rightarrow\left(x \rightarrow 0^{\prime}\right)^{\prime}\right)^{\prime} \rightarrow y \stackrel{(\mathrm{B} .2)}{\approx}\left(x \rightarrow 0^{\prime}\right)^{\prime \prime} \rightarrow y \\
& \stackrel{(\mathrm{B} .2)}{\approx}\left(\left(0^{\prime} \rightarrow x\right) \rightarrow 0^{\prime}\right)^{\prime \prime} \rightarrow y \stackrel{(\mathrm{B} .3)}{\approx}\left(\left(0^{\prime} \rightarrow x\right) \rightarrow 0^{\prime}\right) \rightarrow y \\
& \stackrel{(\mathrm{B} .2)}{\approx}\left(x \rightarrow 0^{\prime}\right) \rightarrow y,
\end{aligned}
$$

Now we have to verify the following weakened version of the identity (B.6):

$$
\left(0 \rightarrow 0^{\prime}\right) \rightarrow x \approx 0^{\prime} \rightarrow x .
$$

Indeed, we have

$$
\begin{aligned}
\left(0 \rightarrow 0^{\prime}\right) \rightarrow x & \stackrel{(\mathrm{B} .4)}{\approx}\left(0^{\prime} \rightarrow 0^{\prime}\right) \rightarrow x \stackrel{(\mathrm{B} .2)}{\approx}\left(\left(0^{\prime} \rightarrow 0^{\prime}\right) \rightarrow 0^{\prime}\right) \rightarrow x \\
& \stackrel{(\mathrm{B} .3)}{\approx}\left(\left(0^{\prime} \rightarrow 0^{\prime}\right) \rightarrow 0^{\prime}\right)^{\prime \prime} \rightarrow x \stackrel{(\mathrm{B} .2)}{\approx}\left(0^{\prime} \rightarrow 0^{\prime}\right)^{\prime \prime} \rightarrow x \\
& \stackrel{(\mathrm{B} .2)}{\approx} 0^{\prime \prime \prime} \rightarrow x \stackrel{\left(\stackrel{(.2)}{\approx} 0^{\prime} \rightarrow x .\right.}{,}
\end{aligned}
$$

Now we are ready to deduct the identities (B.5) and (B.6):

$$
\begin{aligned}
& \text { (B.5) }
\end{aligned}
$$

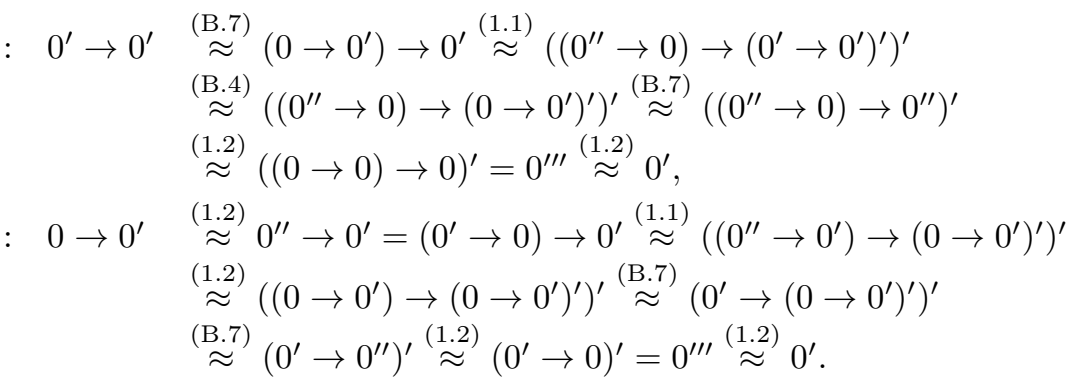

Lemma is proved.

Now we are ready to prove the following assertion that evidently implies Proposition B.1 with $\mathbf{A}=\mathbf{B A}$.

Lemma B.3. A variety of implication zroupoids $\mathbf{X}$ does not contain the variety $\mathbf{B A}$ if and only if $\mathbf{X}$ satisfies the identity

$$
0 \approx 0^{\prime} .
$$

Proof. The "only if" part is evident because $\mathbf{2}_{\mathbf{b}}$ does not satisfy (B.8). Now we verify the "if" part. Arguing a contradiction, we suppose that $\mathbf{X} \nsupseteq \mathbf{B A}$ and $\mathbf{X}$ does not satisfy the identity (B.8). Let $A$ be an algebra in $\mathbf{X}$ such that (B.8) wrong in A. Put $1:=0^{\prime}$. Observe that

$$
0 \rightarrow 1=0 \rightarrow 0^{\prime} \stackrel{(\text { B. } 6)}{=} 1 \stackrel{(\text { B.5) }}{=} 0^{\prime} \rightarrow 0^{\prime}=1 \rightarrow 1 \text { and } 1 \rightarrow 0=0^{\prime} \rightarrow 0=0^{\prime \prime} \stackrel{(1.2)}{=} 0 .
$$

Hence $\langle\{0,1\}, \rightarrow, 0\rangle$ is a subalgebra of $A$ which is isomorphic to $\mathbf{2}_{\mathbf{b}}$, implying that $\mathbf{2}_{\mathrm{b}} \in \mathbf{X}$.

Thus, we have proved Proposition B.1 with $\mathbf{A}=\mathbf{B A}$. This completes the proof of this proposition as a whole. 
Acknowledgements. This work was started when the second and the third authors took part in the Emil Artin International Conference held in Yerevan in MayJune of 2018. The authors are deeply grateful to Professor Yuri Movsisyan and his colleagues for the excellent organization of the conference and the creation of the favorable atmosphere that contributed to the appearance of this article. The authors would like to express also their gratitude to the anonymous referee for his/her valuable remarks and suggestions that contributed to a significant improvement of the original version of the manuscript, in general and to the proof of Theorem 1.1 given in Section 2, in particular.

\section{REFERENCES}

[1] S. Burris, H.P. Sankappanavar, A Course in Universal Algebra, Graduate Texts in Mathematics, 78, Berlin-Heidelberg-N. Y.: Springer-Verlag (1981).

[2] J.M. Cornejo, H.P. Sankappanavar, Semisimple varieties of implication zroupoids, Soft Comput., 20 (2016), 3139-3151.

[3] J.M. Cornejo, H.P. Sankappanavar, On derived algebras and subvarieties of implication zroupoids, Soft Comput., 21 (2017), 6963-6982.

[4] J.M. Cornejo, H.P. Sankappanavar, On implicator groupoids, Algebra Universalis, 77 (2017), $125-146$.

[5] J.M. Cornejo, H.P. Sankappanavar, Implication zroupoids and identities of associative type, Quasigroups and Related Systems, 26 (2018), 13-34.

[6] G. Grätzer, Lattice Theory: Foundation, Basel: Birkhäuser, Springer Basel (2011).

[7] I.I. Mel'nik, The description of some lattices of semigroup varieties, Izv. VUZ. Matem., No. 7 (1972), 65-74 [Russian].

[8] J. Plonka and A. Romanowska, Semilattice sums, A.Romanowska and J.D.H.Smith (eds), Universal Algebra and Quasigroup Theory, Berlin: Heldermann Verlag (1992), 123-158.

[9] H.P. Sankappanavar, De Morgan algebras: New perspectives and applications, Sci. Math. Jpn., 75 (2012), 21-50.

[10] B.M. Vernikov, Special elements in lattices of semigroup varieties, Acta Sci. Math. (Szeged), 81 (2015), 79-109.

[11] M.V. Volkov, Modular elements of the lattice of semigroup varieties, Contrib. General Algebra, 16 (2005), 275-288.

[12] S.L. Wismath, The lattice of varieties and pseudovarieties of band monoids, Semigroup Forum, 33 (1986), 187-198.

S.V. Gusev, B.M. Vernikov: Institute of Natural Sciences and Mathematics, Ural

Federal University, Lenina Str. 51, 620000 EkAterinburg, Russia

E-mail address: sergey.gusb@gmail.com, bvernikov@gmail.com

H.P. Sankappanavar: Department of Mathematics, State University of New York, New Paltz, NY 12561, New York, U.S.A.

E-mail address: sankapph@newpaltz.edu 